\title{
Introduction to the Special Issue on High-Performance and Embedded Architectures and Compilers
}

Papers in the field of computer architecture and compiler design are typically published in conferences and extended versions of them are occasionally published in archival journals. The merit of using conferences as the primary publication vehicle is rapid dissemination, but there are several issues with the conference publication model. First, the review process associated with conferences is not as solid as that of journals: Review loads are often huge and the deadlines are strict, leading to low quality reviews in many cases. Papers with small flaws get rejected and are resubmitted to a different conference and reviewed by a new set of referees because the review history of papers is not maintained. This is an inefficient process. By contrast, articles submitted to journals can have multiple rounds of reviews by the same reviewers. Articles on a clear path to being published can be improved by establishing a dialogue between the authors and the reviewers. This saves time for both authors and reviewers, and hence for the community as a whole. Furthermore, the final version of the article is checked by the reviewers again, and the articles enjoy a copyediting phase before being published, which leads to higher quality publications. Second, articles published in journals are indexed by citation indexes generally accepted across disciplines. This is getting more and more important for many researchers in computer architecture and compiler design.

In a joint venture between $A C M$ TACO and the High-Performance and Embedded Architectures and Compilers Conference (HiPEAC), we decided to try out a new publication model based on a journal-first rather than on a conference-first model of publication. The result of this experiment is this special issue which includes 37 high-quality publications that were evaluated and revised in two review rounds between July 15 and November 15. Thanks to the dialogue between authors and reviewers in the two review rounds, the acceptance rate exceeded $30 \%$ without compromising the quality.

Key to running the fast review process was recruiting a set of around 80 distinguished reviewers - individuals who often participate in program committees of our top conferences. 117 original articles were submitted to ACM TACO on July 15, 2011. In previous years, HiPEAC attracted about 80 submissions so we noted a significant increase in the interest of this new model. The review load per reviewer was strictly limited to 5 papers of 20 pages, and the reviewers got 6 weeks to turn in their reviews. After a first round of reviews, 7 articles were conditionally accepted, while 46 articles had to be revised in a second round. The rest of the articles were rejected. After the second round, another 23 manuscripts were accepted and 7 manuscripts were accepted subject to minor revision. In the end, 37 articles were accepted and another 8 articles were sent into a third round of review and are likely to be accepted by TACO eventually. Reviewers offered lengthy reviews with concrete advice on how the manuscripts could be improved. The whole process with two review rounds took only 4 months to conclude. The experiment has successfully demonstrated that a journal can have turnaround times that are as fast as conferences as well as offer the authors at all of the advantages of a journal publication.

(c) 2012 ACM 1544-3566/2012/01-ART18 $\$ 10.00$

DOI 10.1145/2086696.2086697 http://doi.acm.org/10.1145/2086696.2086697 
Authors of the articles in this special issue have been invited to present their work at HiPEAC 2012 in Paris. Because of the positive experience with this publication model, we will continue to invest in it and refine it based on the experiences gathered in this experiment.

Guest Editors

Per Stenström And Koen De Bosschere 